PROCEEDINGS OF THE

AMERICAN MATHEMATICAL SOCIETY

Volume 129, Number 11, Pages 3425-3435

S 0002-9939(01)05954-8

Article electronically published on April 24, 2001

\title{
VOLUME GROWTH AND PARABOLICITY
}

\author{
ILKKA HOLOPAINEN AND PEKKA KOSKELA
}

(Communicated by Albert Baernstein II)

\begin{abstract}
We characterize $p$-parabolicity of a noncompact complete Riemannian manifold $M$ in terms of the volume growth of $M$ under very weak assumptions on $M$. Some of the results also apply to the setting of metric measure spaces.
\end{abstract}

\section{INTRODUCTION}

In this note we study $p$-parabolicity of metric measure spaces in terms of the volume growth. We mainly concentrate on the case of a noncompact complete Riemannian manifold $M$. To introduce some of our results, we fix a point $o \in M$ and write $V(t)=|B(o, t)|$ for the volume of the geodesic ball of radius $t$ centered at $o$ and $|x|$ for the distance between points $x$ and $o$. It is known that the condition

$$
\int_{1}^{\infty}\left(\frac{t}{V(t)}\right)^{1 /(p-1)} d t=\infty
$$

is always sufficient for the $p$-parabolicity of $M$ but not a necessary condition in general; see for example [H3]. Recall that a Riemannian manifold $M$ is called $p$ parabolic if it does not admit a Green's function for the $p$-Laplace equation. Here, a Green's function $g=g(\cdot, y)$ for the $p$-Laplace equation on $M$ is (if it exists) a certain positive solution of

$$
-\operatorname{div}\left(|\nabla g|^{p-2} \nabla g\right)=\delta_{y}, \quad y \in M,
$$

in the sense of distributions, that is,

$$
\int_{M}\left\langle|\nabla g|^{p-2} \nabla g, \nabla \varphi\right\rangle d m=\varphi(y)
$$

for every $\varphi \in C_{0}^{\infty}(M)$. Equivalently, $M$ is $p$-parabolic if $\operatorname{cap}_{p}(M, C)=0$ for every compact set $C \subset M$; see [H1] and [H2]. Here

$$
\operatorname{cap}_{p}(M, C)=\inf _{u} \int_{M}|\nabla u|^{p} d m
$$

where the infimum is taken over all functions $u \in C_{0}^{\infty}(M)$, with $u \geq 1$ in $C$. The $p$-parabolicity of a metric measure space will be defined in Section 2 .

Received by the editors December 1, 1999 and, in revised form, April 3, 2000.

2000 Mathematics Subject Classification. Primary 58J60, 53C20, 31C12.

Key words and phrases. Volume growth, harmonic function, Green's function, parabolicity.

The first author's work was supported by the Academy of Finland, projects 6355 and 44333.

The second author's work was supported by the Academy of Finland, project 39788. 
It has been an extensively studied problem to find conditions on $M$ in order to characterize $p$-parabolicity of $M$ in terms of the growth of $V(t)$. To be brief, we just refer to [LT] and [S] for the case $p=2$, and to [H3] for all $1<p<\infty$. The recent articles by Grigor'yan [G] and $\mathrm{Li}$ [Li] contain thorough surveys on these and related topics. An interesting open question is whether (1.1) and $p$-parabolicity are equivalent for noncompact complete Riemannian manifolds with nonnegative Ricci curvature outside a compact set. Theorem 1.7 below settles the problem in the case of maximal volume growth.

Let $\gamma$ be a geodesic segment in $M$ between points $o$ and $x$, that is, a minimizing geodesic parametrized by arclength. We say that a volume doubling condition and a weak $(1, p)$-Poincaré inequality hold along $\gamma \mid[a, b]$, where $[a, b] \subset(0,|x|]$, if there exists a constant $c$ such that for every $t \in[a, b]$

$$
|B(\gamma(t), 2 s)| \leq c|B(\gamma(t), s)|,
$$

whenever $0<s \leq t / 4$, and

$$
f_{B_{\gamma}(t)}\left|u-u_{B_{\gamma}(t)}\right| d m \leq c t\left(f_{2 B_{\gamma}(t)}|\nabla u|^{p} d m\right)^{1 / p},
$$

whenever $B_{\gamma}(t)=B(\gamma(t), t / 8)$ and $u \in W^{1, p}\left(2 B_{\gamma}(t)\right)$.

It follows from [H3, §2] that $M$ is not $p$-parabolic if for each $x \in M \backslash B(o, 1)$ there exists a geodesic segment $\gamma$ from $o$ to $x$ such that the doubling condition (1.2) and Poincaré's inequality (1.3) hold along $\gamma \mid[1,|x|]$ and that

$$
\int_{1}^{|x|}\left(\frac{t}{|B(\gamma(t), t / 4)|}\right)^{1 /(p-1)} d t \leq c<\infty
$$

where the constant $c$ is independent of $x$. It is natural to ask whether the existence of a single geodesic ray $\gamma$ emanating from $o$ with

$$
\int_{1}^{\infty}\left(\frac{t}{|B(\gamma(t), t / 4)|}\right)^{1 /(p-1)} d t<\infty
$$

and satisfying the properties (1.2) and (1.3) along $\gamma$ would imply the non-p-parabolicity of $M$. The following theorem answers this question in the affirmative.

1.4. Theorem. Let $M$ be a noncompact, complete Riemannian manifold. Then $M$ admits a Green's function for the $p$-Laplace equation if for each $R>1$ there exists a point $x \in M \backslash B(o, R)$ and a geodesic segment from o to $x$ such that (1.2) and (1.3) hold along $\gamma \mid[1, R]$ and that

$$
\int_{1}^{R}\left(\frac{t}{|B(\gamma(t), t / 4)|}\right)^{1 /(p-1)} d t \leq c<\infty,
$$

where the constant $c$ is independent of $R$.

1.6. Corollary. Suppose that the Ricci curvature of $M^{n}$ satisfies

$$
\operatorname{Ric}(x) \geq \frac{-(n-1) K^{2}}{(1+|x|)^{2}}
$$

for some constant $K \geq 0$. Then $M^{n}$ admits a Green's function for the $p$-Laplace equation if for each $R>1$ there exists a point $x \in M^{n} \backslash B(o, R)$ and a geodesic 
segment from o to $x$ such that

$$
\int_{1}^{R}\left(\frac{t}{|B(\gamma(t), t / 4)|}\right)^{1 /(p-1)} d t \leq c<\infty
$$

where the constant $c$ is independent of $R$.

A straightforward modification of the proof of the Bishop volume comparison theorem [BC, pp. 253-257] implies that $V(t) \leq c_{1} t^{n}$ for all sufficiently large $t$ if $M^{n}$ has nonnegative Ricci curvature outside a compact set. In this case, we say that $M^{n}$ has maximal volume growth if an estimate $V(t) \geq c_{2} t^{n}$ also holds for all sufficiently large $t$. Theorem 1.4 together with the relative Bishop-Gromov volume comparison theorem then implies the following result.

1.7. Theorem. Let $M^{n}$ be a noncompact, complete n-dimensional Riemannian manifold with nonnegative Ricci curvature outside a compact set and maximal volume growth. Then $M^{n}$ is p-parabolic if and only if $p \geq n$.

We obtain our results by combining volume comparison techniques with a lower bound for a certain variational capacity. The proof of the crucial capacity estimate is based on a chaining argument, where a certain doubling condition and Poincaré's inequality play a dominant role. In fact, we can carry over the argument for the capacity estimate not only in a Riemannian setting but also on metric spaces (see Theorem 2.10).

Throughout the paper $c$ will be a generic positive constant whose actual value may vary, even within a line.

\section{Main estimates}

Let $(X, d)$ be a metric space and let $\mu$ be a Borel regular measure on $X$ such that each open ball of $X$, denoted by $B(x, r)$, has a positive and finite measure. We write $|A|=\mu(A)$ if $A$ is a measurable set in $X$. Recall from [HK1] and [HK2] that a Borel function $\rho: X \rightarrow[0, \infty]$ is called an upper gradient of a real-valued function $u$ in $X$ if

$$
|u(a)-u(b)| \leq \int_{\gamma} \rho d s
$$

for each rectifiable curve $\gamma$ in $X$ with end points $a$ and $b$. Note that $\rho=|\nabla u|$ is an upper gradient of a smooth function $u$ on a Riemannian manifold. As in [HK2], we let $\mathcal{S}$ be any of the following three classes of real-valued functions defined on measurable subsets of $X$ : Lipschitz, continuous, or measurable. A triple $(E, F ; U)$ is called a condenser if $E$ and $F$ are disjoint closed subsets of an open set $U \subset X$. For $1 \leq p<\infty$, we define the $(p, \mathcal{S})$-capacity $\operatorname{cap}_{p}^{\mathcal{S}}(E, F ; U)$ of the condenser $(E, F ; U)$ by

$$
\operatorname{cap}_{p}^{\mathcal{S}}(E, F ; U)=\inf \int_{U} \rho^{p} d \mu,
$$

where the infimum is taken over all upper gradients $\rho$ of all functions $u$ in $U$ that belong to $\mathcal{S}$ and satisfy $u \mid E \geq 1$ and $u \mid F \leq 0$. Such a function $u$ is called admissible for $(E, F ; U)$. If $U \subset X$ is open and $C \subset U$ is compact, we set

$$
\operatorname{cap}_{p}^{\mathcal{S}}(U, C)=\inf \int_{X} \rho^{p} d \mu
$$


where the infimum is taken over all upper gradients $\rho$ of all compactly supported functions $u$ in $U$ that belong to $\mathcal{S}$ and satisfy $u \mid C \geq 1$. Also here $u$ is called admissible for $(U, C)$. If $\operatorname{cap}_{p}^{\mathcal{S}}(X, C)=0$ for all compact sets $C$ in $X$, we say that $X$ is $(p, \mathcal{S})$-parabolic. The following lemma is well known for the usual $p$-capacity in $\mathbb{R}^{n}$.

2.1. Lemma. Let $C_{i}, i=1,2, \ldots$, be a compact subset of an open set $\Omega_{i}$ in $X$. If $C_{1} \subset \Omega_{1} \subset C_{2} \subset \Omega_{2} \subset \cdots \subset \Omega=\bigcup_{i} \Omega_{i}$ and $1<p<\infty$, then

$$
\operatorname{cap}_{p}^{\mathcal{S}}\left(\Omega, C_{1}\right) \leq\left(\sum_{i=1}^{\infty} \operatorname{cap}_{p}^{\mathcal{S}}\left(\Omega_{i}, C_{i}\right)^{1 /(1-p)}\right)^{1-p} .
$$

Proof. If $\operatorname{cap}_{p}^{\mathcal{S}}\left(\Omega_{i}, C_{i}\right)=0$ for some $i$, then also $\operatorname{cap}_{p}^{\mathcal{S}}\left(\Omega, C_{1}\right)=0$, and the estimate (2.2) holds. Thus we may assume that $\operatorname{cap}_{p}^{\mathcal{S}}\left(\Omega_{i}, C_{i}\right)>0$ for all $i$. Fix $\varepsilon>0$. For each $i$ choose a function $u_{i} \in \mathcal{S}$, with compact support in $\Omega_{i}$ and with $u_{i} \mid C_{i}=1$, and an upper gradient $\rho_{i}$ of $u_{i}$ such that

$$
\int_{\Omega_{i}} \rho_{i}^{p} d \mu \leq \operatorname{cap}_{p}^{\mathcal{S}}\left(\Omega_{i}, C_{i}\right)+\varepsilon
$$

We may choose the upper gradients $\rho_{i}$ so that the sets where $\rho_{i} \neq 0$ are pairwise disjoint. Let $a_{i}$ be a sequence of nonnegative numbers with $\sum_{i=1}^{j} a_{i}=1$. We define

$$
v=\sum_{i=1}^{j} a_{i} u_{i}
$$

and

$$
\rho=\sum_{i=1}^{j} a_{i} \rho_{i}
$$

Then $v$ is admissible for $\left(\Omega, C_{1}\right)$ and $\rho$ is an upper gradient of $v$. Since the sets spt $\rho_{i}$ are mutually disjoint, we have

$$
\begin{aligned}
\operatorname{cap}_{p}^{\mathcal{S}}\left(\Omega, C_{1}\right) & \leq \int_{\Omega} \rho^{p} d \mu \\
& =\sum_{i=1}^{j} a_{i}^{p} \int_{\Omega_{i}} \rho_{i}^{p} d \mu \\
& \leq \sum_{i=1}^{j} a_{i}^{p} \operatorname{cap}_{p}^{\mathcal{S}}\left(\Omega_{i}, C_{i}\right)+\varepsilon
\end{aligned}
$$

Hence

$$
\operatorname{cap}_{p}^{\mathcal{S}}\left(\Omega, C_{1}\right) \leq \sum_{i=1}^{j} a_{i}^{p} \operatorname{cap}_{p}^{\mathcal{S}}\left(\Omega_{i}, C_{i}\right) .
$$

The claim now follows by choosing

$$
a_{i}=\operatorname{cap}_{p}^{\mathcal{S}}\left(\Omega_{i}, C_{i}\right)^{1 /(1-p)}\left(\sum_{k=1}^{j} \operatorname{cap}_{p}^{\mathcal{S}}\left(\Omega_{k}, C_{k}\right)^{1 /(1-p)}\right)^{-1}
$$

and letting $j \rightarrow \infty$.

Recall that a metric space $X$ is called proper if its closed balls are compact. 
2.3. Proposition. Let $X$ be a proper metric space with $\operatorname{diam} X=\infty$. Then $X$ is $(p, \mathcal{S})$-parabolic, with $1<p<\infty$, if

$$
\int_{1}^{\infty}\left(\frac{t}{V(t)}\right)^{1 /(p-1)} d t=\infty
$$

where $V(t)=|B(o, t)|$ for a fixed $o \in X$.

Proof. Let $C$ be an arbitrary compact set in $X$. Choose $r>0$ such that $C \subset \bar{B}(o, r)$ and write $r_{i}=2^{i} r$ for $i=0,1, \ldots$ By Lemma 2.1,

$$
\begin{aligned}
\operatorname{cap}_{p}^{\mathcal{S}}(X, C) & \leq \operatorname{cap}_{p}^{\mathcal{S}}(X, \bar{B}(o, r)) \\
& \leq\left(\sum_{i=0}^{\infty} \operatorname{cap}_{p}^{\mathcal{S}}\left(B\left(o, r_{i+1}\right), \bar{B}\left(o, r_{i}\right)\right)^{1 /(1-p)}\right)^{1-p} .
\end{aligned}
$$

Fix $\varepsilon \in] o, r\left[\right.$ and define a function $u_{i}$ by

$$
u_{i}(x)= \begin{cases}1, & |x| \leq r_{i} ; \\ \frac{r_{i+1}-\varepsilon-|x|}{r_{i}-\varepsilon}, & r_{i}<|x|<r_{i+1}-\varepsilon ; \\ 0, & |x| \geq r_{i+1}-\varepsilon .\end{cases}
$$

Then the function $\rho_{i}$,

$$
\rho_{i}(x)= \begin{cases}\left(r_{i}-\varepsilon\right)^{-1}, & r_{i}<|x|<r_{i+1}-\varepsilon \\ 0, & \text { elsewhere }\end{cases}
$$

is an upper gradient of $u_{i}$. Since $u_{i}$ is admissible for $\left(B\left(o, r_{i+1}\right), \bar{B}\left(o, r_{i}\right)\right)$, we get by letting $\varepsilon \rightarrow 0$ that

$$
\operatorname{cap}_{p}^{\mathcal{S}}\left(B\left(o, r_{i+1}\right), \bar{B}\left(o, r_{i}\right)\right) \leq\left|B\left(o, r_{i+1}\right)\right| r_{i}^{-p} .
$$

Substituting this into (2.5) and using (2.4) yields $\operatorname{cap}_{p}^{\mathcal{S}}(X, C)=0$.

2.6. Definition. Let $(E, F ; U)$ be a condenser and let $\lambda \geq 1$. We say that a sequence of balls $B_{i}=B\left(x_{i}, r_{i}\right), i=0,1, \ldots, k$, is a chain connecting $E$ and $F$ in $U$ if

(1) $B_{0} \subset E$ and $B_{k} \subset F$;

(2) $B_{i} \cap B_{i+1} \neq \emptyset$ for every $i=0, \ldots, k-1$;

(3) $\lambda B_{i} \subset U$ for every $i=1, \ldots, k$; and

(4) there exists a constant $C_{o l}$ such that no point of $X$ belongs to more than $C_{o l}$ balls $\lambda B_{i}$.

We use the notion of a chain connecting $E$ and $F$ in $U$ to estimate $\operatorname{cap}_{p}^{\mathcal{S}}(E, F ; U)$ from below. For that purpose, let

$$
c_{d, i}=\frac{\left|B_{i} \cup B_{i+1}\right|}{\left|B_{i} \cap B_{i+1}\right|} .
$$

Furthermore, suppose that $c_{p, i}$ is a constant such that the $(1, p)$-Poincaré inequality

$$
f_{B_{i}}\left|u-u_{B_{i}}\right| d \mu \leq c_{p, i} r_{i}\left(f_{\lambda B_{i}} \rho^{p} d \mu\right)^{1 / p}
$$


holds for each $\mathcal{S}$ function $u$ in $\lambda B_{i}$ and for each upper gradient $\rho$ of $u$ in $\lambda B_{i}$. Finally, we set

$$
c_{i}= \begin{cases}c_{d, 0} c_{p, 0}, & \text { if } i=0 \\ \left(c_{d, i-1}+c_{d, i}\right) c_{p, i}, & \text { if } i=1, \ldots, k-1 \\ c_{d, k-1} c_{p, k}, & \text { if } i=k .\end{cases}
$$

2.10. Theorem. Let $(E, F ; U)$ be a condenser, and $B_{i}=B\left(x_{i}, r_{i}\right), i=0,1, \ldots, k$, a chain connecting $E$ and $F$ in $U$ with $\lambda \geq 1$. If $1<p<\infty$, then

$$
\operatorname{cap}_{p}^{\mathcal{S}}(E, F ; U) \geq \frac{1}{C_{o l}}\left(\sum_{i=0}^{k}\left(\frac{c_{i}^{p} r_{i}^{p}}{\left|\lambda B_{i}\right|}\right)^{1 /(p-1)}\right)^{1-p},
$$

where the constants $c_{i}$ are given by (2.9). Furthermore,

$$
\operatorname{cap}_{1}^{\mathcal{S}}(E, F ; U) \geq \frac{1}{C_{o l}} \min \left\{\left|\lambda B_{0}\right| /\left(c_{0} r_{0}\right), \ldots,\left|\lambda B_{k}\right| /\left(c_{k} r_{k}\right)\right\}
$$

Proof. Let $u$ be an arbitrary $\mathcal{S}$ function in $U$ with $u \mid E \geq 1$ and $u \mid F \leq 0$. By the triangle inequality,

$$
1 \leq \sum_{i=0}^{k-1}\left|u_{B_{i}}-u_{B_{i+1}}\right| \leq \sum_{i=0}^{k-1}\left(\left|u_{B_{i}}-u_{K_{i}}\right|+\left|u_{K_{i}}-u_{B_{i+1}}\right|\right),
$$

where $K_{i}=B_{i} \cap B_{i+1}$. If $\rho$ is an arbitrary upper gradient of $u$ in $U$, we have by (2.7) and (2.8) that

$$
\begin{aligned}
\left|u_{B_{i}}-u_{K_{i}}\right| & =\left|f_{K_{i}}\left(u_{B_{i}}-u\right) d \mu\right| \\
& \leq f_{K_{i}}\left|u-u_{B_{i}}\right| d \mu \\
& \leq \frac{\left|B_{i}\right|}{\left|K_{i}\right|} f_{B_{i}}\left|u-u_{B_{i}}\right| d \mu \\
& \leq c_{d, i} f_{B_{i}}\left|u-u_{B_{i}}\right| d \mu \\
& \leq c_{d, i} c_{p, i} r_{i}\left(f_{\lambda B_{i}} \rho^{p} d \mu\right)^{1 / p}
\end{aligned}
$$

and, similarly,

$$
\left|u_{K_{i}}-u_{B_{i+1}}\right| \leq c_{d, i} c_{p, i+1} r_{i+1}\left(f_{\lambda B_{i+1}} \rho^{p} d \mu\right)^{1 / p} .
$$


Suppose first that $1<p<\infty$. By combining the estimates (2.11)-(2.13) and using Hölder's inequality and the overlapping condition (4), we obtain that

$$
\begin{aligned}
1 & \leq \sum_{i=0}^{k} c_{i} r_{i}\left|\lambda B_{i}\right|^{-1 / p}\left(\int_{\lambda B_{i}} \rho^{p} d \mu\right)^{1 / p} \\
& \leq\left(\sum_{i=0}^{k} \int_{\lambda B_{i}} \rho^{p} d \mu\right)^{1 / p}\left(\sum_{i=0}^{k}\left(\frac{c_{i}^{p} r_{i}^{p}}{\left|\lambda B_{i}\right|}\right)^{1 /(p-1)}\right)^{(p-1) / p} \\
& \leq\left(C_{o l} \int_{U} \rho^{p} d \mu\right)^{1 / p}\left(\sum_{i=0}^{k}\left(\frac{c_{i}^{p} r_{i}^{p}}{\left|\lambda B_{i}\right|}\right)^{1 /(p-1)}\right)^{(p-1) / p}
\end{aligned}
$$

The case $p=1$ follows similarly but without appealing to Hölder's inequality.

To obtain a lower bound for $\operatorname{cap}_{p}^{\mathcal{S}}(X, C)$, we define a notion of a chain to infinity as follows.

2.14. Definition. Let $X$ be a proper metric space with $\operatorname{diam} X=\infty$. We say that a sequence of balls $B_{i}=B\left(x_{i}, r_{i}\right) \subset X, i=0,1, \ldots$, is a chain to infinity, with $\lambda \geq 1$, if

(i) $B_{i} \rightarrow \infty$ as $i \rightarrow \infty$;

(ii) $B_{i} \cap B_{i+1} \neq \emptyset$ for every $i$; and

(iii) there exists a constant $C_{o l}$ such that no point of $X$ belongs to more than $C_{o l}$ balls $\lambda B_{i}$.

Above $B_{i} \rightarrow \infty$ means that, for any compact set $K \subset X, B_{i} \subset X \backslash K$ as soon as $i$ is sufficiently large.

We define the constants $c_{d, i}$ and $c_{p, i}$ as in (2.7) and (2.8), respectively, and then we set

$$
c_{i}= \begin{cases}c_{d, 0} c_{p, 0}, & \text { if } i=0 \\ \left(c_{d, i-1}+c_{d, i}\right) c_{p, i}, & \text { otherwise }\end{cases}
$$

Now the counterpart for Theorem 2.10 reads as follows.

2.16. Theorem. Suppose that $X$ is proper with $\operatorname{diam} X=\infty$. Let $\left\{B_{i}\right\}$ be a chain to infinity with $\lambda \geq 1$, and let $c_{i}$ be given by (2.15). If $1<p<\infty$ and

$$
\sum_{i=0}^{\infty}\left(\frac{c_{i}^{p} r_{i}^{p}}{\left|\lambda B_{i}\right|}\right)^{1 /(p-1)}=S<\infty
$$

then

$$
\operatorname{cap}_{p}^{\mathcal{S}}\left(X, \bar{B}_{0}\right) \geq \frac{1}{C_{o l} S^{p-1}}>0 .
$$

If

$$
\frac{c_{i} r_{i}}{\left|\lambda B_{i}\right|}<c<\infty
$$

for every $i$, then

$$
\operatorname{cap}_{1}^{\mathcal{S}}\left(X, \bar{B}_{0}\right) \geq c>0
$$




\section{Applications to the Riemannian Setting}

In this section we give some examples in order to apply Theorem 2.10 and eventually prove the results mentioned in the introduction. Theorem 1.4 is an immediate consequence of the following.

3.1. Theorem. Let $R>r>0$. Suppose that there exist a point $x \in M \backslash B(o, 2 R)$ and a segment $\gamma$ from o to $x$ such that (1.2) and (1.3) hold along $\gamma \mid[r / 2,2 R]$. Then

$$
\operatorname{cap}_{p}(B(o, R), \bar{B}(o, r)) \geq c\left(\int_{r / 2}^{2 R}\left(\frac{t}{|B(\gamma(t), t / 4)|}\right)^{1 /(p-1)} d t\right)^{1-p} .
$$

In particular, if

$$
V(t) \leq c|B(\gamma(t), t / 4)|
$$

for each $t \in[r / 2,2 R]$, then

$$
\operatorname{cap}_{p}(B(o, R), \bar{B}(o, r)) \geq c\left(\int_{r / 2}^{2 R}\left(\frac{t}{V(t)}\right)^{1 /(p-1)} d t\right)^{1-p} .
$$

Proof. In order to apply Theorem 2.10 we first construct a chain connecting $\bar{B}(o, r)$ and $M \backslash B(o, R)$ in $M$ with parameter $\lambda=2$. For each integer $i \geq 0$, we write $t_{i}=(9 / 8)^{i-1} r$. Let $k$ be an integer such that $7 t_{k} / 8 \geq R$ and $t_{k+1} \leq 2 R$. Then $B\left(\gamma\left(t_{0}\right), t_{0} / 8\right) \subset B(o, r)$ and $B\left(\gamma\left(t_{k}\right), t_{k} / 8\right) \subset M \backslash B(o, R)$. Also the conditions (2)(4) in Definition 2.6 hold with $\lambda=2$. Thus the sequence of balls $B_{i}=B\left(\gamma\left(t_{i}\right), t_{i} / 8\right)$, with $i=0, \ldots, k$, is a desired chain. Furthermore,

$$
B\left(\gamma\left(15 t_{i+1} / 16\right), t_{i+1} / 16\right) \subset B_{i} \cap B_{i+1}
$$

and

$$
B_{i} \cup B_{i+1} \subset B\left(\gamma\left(15 t_{i+1} / 16\right), 3 t_{i+1} / 16\right) .
$$

Let $c_{d, i}, c_{p, i}$, and $c_{i}$ be given by (2.7), (2.8), and (2.9), respectively. Then

$$
c_{d, i}=\frac{\left|B_{i} \cup B_{i+1}\right|}{\left|B_{i} \cap B_{i+1}\right|} \leq c
$$

by the doubling condition (1.2). Since $c_{p, i} \leq c$ by (1.3), we have $c_{i} \leq c$. Theorem 2.10 then implies that

$$
\operatorname{cap}_{p}(B(o, R), \bar{B}(o, r)) \geq c\left(\sum_{i=0}^{k}\left(\frac{t_{i}^{p}}{\left|2 B_{i}\right|}\right)^{1 /(p-1)}\right)^{1-p} .
$$

On the other hand,

$$
|B(\gamma(t), t / 4)| \leq c\left|2 B_{i}\right|
$$

for every $t \in\left[t_{i}, t_{i+1}\right]$ again by (1.2). Hence

$$
\frac{t_{i}^{p}}{\left|2 B_{i}\right|} \leq c\left(\int_{t_{i}}^{t_{i+1}}\left(\frac{t}{|B(\gamma(t), t / 4)|}\right)^{1 /(p-1)} d t\right)^{p-1},
$$


and so

$$
\operatorname{cap}_{p}(B(o, R), \bar{B}(o, r)) \geq c\left(\int_{t_{0}}^{t_{k+1}}\left(\frac{t}{|B(\gamma(t), t / 4)|}\right)^{1 /(p-1)} d t\right)^{1-p}
$$

which implies (3.2). The estimate (3.3) follows immediately from (3.2) if $V(t) \leq$ $c|B(\gamma(t), t / 4)|$ for every $t \in[r / 2,2 R]$.

3.4. Example. Suppose that the Ricci curvature of $M^{n}$ satisfies the estimate

$$
\operatorname{Ric}(x) \geq \frac{-(n-1) K^{2}}{(1+|x|)^{2}}
$$

for some constant $K \geq 0$. Let $\gamma$ be a segment from $o$ to some point $x$. It follows from the well-known Bishop-Gromov comparison theorem (see [BC] and [CGT]) and, respectively, from Buser's isoperimetric inequality $[\mathrm{B}]$ that the volume doubling condition (1.2) and, respectively, the Poincaré inequality (1.3) hold along $\gamma$; see [H3, Example 2.20] for details. In fact, the Poincaré inequality holds with the same balls on both sides of the inequality.

Thus we obtain the following consequence of Theorem 3.1 ,

3.6. Corollary. Suppose that the Ricci curvature of $M^{n}$ satisfies (3.5). Let $R>$ $r>0$ and let $\gamma$ be any segment from o to a point $x \in M^{n} \backslash B(o, 2 R)$. Then

$$
\operatorname{cap}_{p}(B(o, R), \bar{B}(o, r)) \geq c\left(\int_{r / 2}^{2 R}\left(\frac{t}{|B(\gamma(t), t / 4)|}\right)^{1 /(p-1)} d t\right)^{1-p} .
$$

Furthermore, if

$$
V(t) \leq c|B(\gamma(t), t / 4)|
$$

for each $t \in[r / 2,2 R]$, then

$$
\operatorname{cap}_{p}(B(o, R), \bar{B}(o, r)) \geq c\left(\int_{r / 2}^{2 R}\left(\frac{t}{V(t)}\right)^{1 /(p-1)} d t\right)^{1-p} .
$$

3.9. Example. Suppose that the Ricci curvature of $M^{n}$ is nonnegative outside a compact set. In particular, the curvature estimate (3.5) holds with some $K$. By scaling the metric we may assume that $M^{n}$ has nonnegative Ricci curvature outside a ball $B(o, 1)$. Observe that the scaling of the metric has no effect on the $p$-parabolicity of $M^{n}$. As in [LT] (and [Liu]), we cover $\partial B(o, 2)$ by closed sets $U_{1}, \ldots, U_{m} \subset \partial B(o, 2)$ with $\operatorname{diam} U_{i}<2$. Let $K_{i}$ be the set of all points $x \in M^{n} \backslash B(o, 2)$ for which there exists a geodesic segment from $o$ to $x$ intersecting $U_{i}$. If $x, y \in K_{i}$, then no geodesic segment from $x$ to $y$ intersects $B(o, 1)$ by the triangle inequality. Since $M^{n} \backslash B(o, 2) \subset \bigcup_{i=1}^{m} K_{i}$, we have

$$
\liminf _{t \rightarrow \infty} \frac{\sum_{i=1}^{m}\left|K_{i} \cap B(o, t)\right|}{V(t)} \geq 1 .
$$

Here the fact $\left|M^{n}\right|=\infty$ from [CGT, Theorem 4.9] was also used. Suppose that

$$
\liminf _{t \rightarrow \infty} \frac{\left|K_{k} \cap B(o, t)\right|}{V(t)}>0
$$


for some $k \in\{1, \ldots, m\}$. Then there exist $c>0$ and $R_{0}>2$ such that

$$
\left|K_{k} \cap B(o, t)\right| \geq c V(t)
$$

for every $t \geq R_{0}$. Let $y$ be any point of $K_{k}$, with $|y|=t \geq R_{0}$. Since no geodesic segment from $y$ to any other point in $K_{k} \cap B(o, t)$ intersects $B(o, 1)$ and $M^{n}$ has nonnegative Ricci curvature outside $B(o, 1)$, the Bishop-Gromov comparison theorem (see [CGT]) and (3.11) imply that

$$
|B(y, t / 4)| \geq c(n)\left|K_{k} \cap B(o, t)\right| \geq c V(t),
$$

where $c$ depends only on $n$ and $m$. In particular, (3.7) holds for all $s>R_{0}$ and all geodesic segments from $o$ to any point $x \in K_{k} \backslash B(o, s)$. Thus we have:

3.12. Lemma. Suppose that the Ricci curvature of $M^{n}$ is nonnegative in $M^{n} \backslash$ $B(o, 1)$ and that (3.10) holds for some $K_{k}$. Then there exist constants $c$ and $R_{0}>2$ such that, for each $s>R_{0}$, there exists a geodesic segment $\gamma$ from o to a point $x \in M^{n} \backslash B(o, s)$, with

$$
V(t) \leq c|B(\gamma(t), t / 4)|
$$

for all $t \in\left[R_{0},|x|\right]$. Furthermore, for $R>r>2 R_{0}$,

$$
\operatorname{cap}_{p}(B(o, R), \bar{B}(o, r)) \geq c\left(\int_{r / 2}^{2 R}\left(\frac{t}{V(t)}\right)^{1 /(p-1)} d t\right)^{1-p} .
$$

In particular,

$$
\operatorname{cap}_{p}(M, \bar{B}(o, r)) \geq c\left(\int_{r / 2}^{\infty}\left(\frac{t}{V(t)}\right)^{1 /(p-1)} d t\right)^{1-p} .
$$

3.13. Lemma. Suppose that $M^{n}$ has nonnegative Ricci curvature in $M^{n} \backslash B(o, 1)$ and maximal volume growth. Then (3.10) holds for some $K_{k}$.

Proof. By slightly modifying the argument in $[\mathrm{BC}$ pp. 253-257] we deduce that

$$
t \mapsto \frac{\left|K_{i} \cap B(o, t)\right|}{(t-1)^{n}-1}
$$

is nonincreasing for all sufficiently large $t$ and all $i$. Hence the limit

$$
\lim _{t \rightarrow \infty} \frac{\left|K_{i} \cap B(o, t)\right|}{t^{n}}
$$

exists for all $i$. Since $M^{n}$ has maximal volume growth, at least one of these limits has to be positive.

We close this paper by observing that Theorem 1.7 now follows from Lemmata 3.12 and 3.13 since

$$
\int_{1}^{\infty}\left(\frac{t}{V(t)}\right)^{1 /(p-1)} d t<\infty
$$

if and only if $1<p<n$ by the volume growth assumption $V(t) \approx t^{n}$. 


\section{REFERENCES}

[BC] R. Bishop and R. Crittenden, Geometry of Manifolds, Academic Press, New York and London, 1964. MR 29:6401

[B] P. Buser, A note on the isoperimetric constant, Ann. Sci. Ecole Norm. Sup. 15 (1982), 213-230. MR 84e:58076

[CGT] J. Cheeger, M. Gromov, and M. Taylor, Finite propagation speed, kernel estimates for functions of the Laplace operator, and the geometry of complete Riemannian manifolds, J. Differential Geometry 17 (1982), 15-53. MR 84b:58109

[G] A. Grigor'yan, Analytic and geometric background of recurrence and non-explosion of the Brownian motion on Riemannian manifolds, Bull. Amer. Math. Soc. 36 (1999), 135-249. MR 99k:58195

[HK1] J. Heinonen and P. Koskela, Quasiconformal maps in metric spaces with controlled geometry, Acta Math. 181 (1998), 1-61. MR 99j:30025

[HK2] J. Heinonen and P. Koskela, A note on Lipschitz functions, upper gradients, and the Poincaré inequality, New Zealand J. Math. 28 (1999), 37-42. MR 2000d:46041

[H1] I. Holopainen, Nonlinear potential theory and quasiregular mappings on Riemannian manifolds, Ann. Acad. Sci. Fenn. Ser. A I Math. Diss. 74 (1990), 1-45. MR 91e:31029

[H2] I. Holopainen, Positive solutions of quasilinear elliptic equations on Riemannian manifolds, Proc. London Math. Soc. (3) 65 (1992), 651-672. MR 94d:58161

[H3] I. Holopainen, Volume growth, Green's functions, and parabolicity of ends, Duke Math. J. 97 (1999), 319-346. MR 2000i:58066.

[Li] P. Li, Curvature and function theory on Riemannian manifolds, Surveys in Diff. Geom. (to appear).

[LT] P. Li and L.F. Tam, Green's functions, harmonic functions, and volume comparison, J. Differential Geom. 41 (1995), 277-318. MR 96f:53054

[Liu] Z. Liu, Ball covering property and nonnegative Ricci curvature outside a compact set, Differential Geometry: Riemannian Geometry, Proc. Symp. Pure Math., vol. 54 (3), Amer. Math. Soc., Providence, RI, 1993, pp. 459-464.

[S] C.-J. Sung, A note on the existence of positive Green's function, J. Funct. Anal. 156 (1998), 199-207. MR 99g:53046

Department of Mathematics, University of Helsinki, P.O. Box 4 (Yliopistonkatu 5), FIN-00014 Helsinki, Finland

E-mail address: ilkka.holopainen@helsinki.fi

Department of Mathematics, University of Jyväskylä, P.O. Box 35, FIN-40351 JYVÄSKYLÄ, FINLAND

E-mail address: pkoskela@math.jyu.fi 